\title{
Un enfoque multidimensional sobre los intermediarios laborales en el medio agrícola
}

\author{
A multidimensional approach to labor intermediaries in agricultural areas
}

\author{
Kim Sánchez SALdaña \\ Área de Antropología, UAEM, México \\ kimsa1910@yahoo.com.mx
}

Recibido: 16.06 .2011

Aprobado definitivamente: 13.12 .2011

\section{RESUMEN}

En este documento se sostiene que el análisis de los intermediarios laborales en el medio rural exige explorar no sólo sus funciones económicas en la movilización y control de la fuerza de trabajo, sino también sus funciones sociales y culturales en su interacción con empleadores y jornaleros agrícolas.

Estas reflexiones surgen del análisis de una clase particular de intermediarios laborales, los capitanes, quienes se encargan de reclutar jornaleros temporales y organizar cuadrillas para la cosecha de hortalizas en el valle de Cuautla, en el estado de Morelos en México. La intervención de los capitanes es crucial en el control de este mercado de trabajo estacional compuesto por comunidades indígenas nahuas, mixtecas y tlapanecas, por lo que se han vuelto imprescindibles para los productores. En ese contexto, la eficacia social de las funciones económicas intermediarias de los capitanes, dependen de su desempeño como intermediarios culturales, cuyo lugar y función se ubica en el espacio fronterizo de los ejes mestizo/indígena y local/foráneo.

A partir de este estudio de caso se propone considerar a otros intermediarios laborales bajo un enfoque multidimensional, pues abre nuevas perspectivas para el análisis de las complejas articulaciones entre comunidades abastecedoras de mano de obra y el sector de la agricultura comercial capitalista, máxime cuando estos ámbitos laborales son nichos migratorios y espacios privilegiados de relaciones interétnicas.

Palabras Clave: Intermediarios agrícolas, intermediación cultural, jornaleros, trabajo agrícola, enclaves de agricultura global

\begin{abstract}
This paper argues that analysis of labor intermediaries in rural areas requires explore not only their economic functions in the mobilization and control of the workforce, but also its social and cultural functions in their interaction with employers and farm laborers. These reflections arise from the analysis of a particular kind of labor intermediaries, the captains, who are responsible for recruiting temporary workers and organize crews to harvest vegetables in the Valley of Cuautla, in the state of Morelos in Mexico. The intervention of the captains are
\end{abstract}


crucial in controlling the seasonal labor market composed of indigenous Nahua, Mixtec and Tlapanecas, so have become essential for producers. In this context, the social efficiency of financial intermediary functions of the captains depend on his performance as cultural intermediaies, whose place and function is located in the border area of the axes mestizo/indigenous and local/foreign. From this case of study, it is proposed to consider other labour intermediaries based on a multidimensional approach, since it opens new perspectives for analyzing the complex links between communities supplying labor and industry of capitalist commercial agriculture, particularly since these areas are spaces for migrant labor opportunities and privileged spaces for inter-ethnic relations.

KEYwORDs: Agricultural intermediaries, cultural intermediation, laborers, farm labor, global agricultural enclaves.

\section{SUMARIO}

1. Introducción. 2. Sistemas de contratación e intermediarios tradicionales. 3. Mediadores e intermediarios culturales. 4. Los capitanes de tenextepango, morelos. 5. Algunas reflexiones comparativas y generales.

\section{SUMMARY}

1. Introduction. 2. Contracting systems and traditional intermediaries. 3. Mediators and cultural intermediaries.

4. "Los capitanes" of Tenextepango, Morelos. 5. Some comparative and general reflections. 


\section{INTRODUCCIÓN}

La presencia de intermediarios en la movilización de la fuerza de trabajo es un fenómeno recurrente en el medio agrícola y, al parecer, un aspecto clave para comprender cómo se estructuran y funcionan estos mercados laborales. Su papel cobra mayor significación cuando vemos que muchos de estos intermediarios están, a su vez, ligados a la contratación de mano de obra migrante y constituyen un puente privilegiado de acceso de ésta a sus potenciales empleadores.

Las razones funcionales de los sistemas de intermediación para cubrir las peculiares necesidades de aprovisionamiento y regulación de la fuerza de trabajo en la explotación agrícola, parecen deducirse por sí solas. Sin embargo, la presencia de instituciones o agentes intermediarios no sólo han sido una respuesta económica a una "dislocación" entre los ámbitos de oferta y demanda de trabajo, sino que también han estado asociados con tareas de control social, en tanto instrumento para disciplinar la mano de obra y contener posibles conflictos laborales. Funciones económicas y funciones políticas han ido de la mano en la historia de los sistemas de intermediación laboral, minando la capacidad de negociación de los trabajadores agrícolas y convirtiéndose así en un mecanismo adicional de acumulación del capital.

En el pasado a estos intermediarios se les conoció como "enganchadores" y hasta nuestros días el término se ha utilizado más ampliamente para identificar no sólo la presencia de sistemas de contratación indirecta basados en el pago por adelantado, sino a otras variantes de reclutamiento y tutela de la fuerza de trabajo que usan múltiples vínculos de dependencia a esas fuentes de empleo. De allí que la persistencia y readecuación de estos métodos para movilizar, retener y expulsar a la mano de obra se adecue ventajosamente a las actuales tendencias de flexibilización y precarización del trabajo que predominan en los enclaves de agricultura intensiva y en la conformación de esas cadenas globales de mercancías.

Dentro del conjunto de los sistemas de contratación indirecta interesa focalizar sobre aquellos que se denominan intermediarios tradicionales, que en países como México denotan a sujetos conocidos como enganchadores, mayordomos, capitanes, cabos o simplemente contratistas. ¿Mediante qué recursos y mecanismos estos intermediarios influyen en la definición de esos regímenes de control de la fuerza laboral en el proceso productivo y en la relación de los jornaleros con sus espacios de trabajo? Nuestro argumento central es que para comprender estas cuestiones es importante revisar las dimensiones sociales y culturales que acompañan al fenómeno del intermediarismo en el ámbito laboral. Asimismo, creemos que un enfoque multidimensional arroja luz sobre estos espacios mediadores que explican el carácter ambiguo y dinámico de su relación con los propios trabajadores.

Para ello proponemos hacer una breve referencia a las características de estos actores en los sistemas de contratación y, en seguida, sobre los fenómenos de mediación en general. A través de un estudio de caso, sobre cierto tipo de intermediarios laborales vinculados a la cosecha de hortalizas en el estado de Morelos en México, pretendemos argumentar la pertinencia de nuestro enfoque y sugerir la manera en que se manifiesta su desempeño en contextos caracterizados por complejos migratorios y relaciones interétnicas. Finalmente, sugerimos que de esta propuesta se pueden desprender algunos criterios de análisis sobre otras figuras intermediarias en mercados laborales análogos.

\section{SISTEMAS DE CONTRATACIÓN E INTERMEDIARIOS TRADICIONALES}

El término de intermediario tradicional se refiere, básicamente, a aquellos individuos que en países como México reúnen las siguientes características: viven en comunidades rurales; se encargan de reunir a la mano de obra en sus lugares de origen y transportarla a las zonas donde se requiere; financian o gestionan el traslado; entregan con frecuencia algún tipo de recursos por adelantado; cumplen generalmente la función de capataces en las labores agrícolas en que se emplea esa mano de obra; cobran comisiones a los productores por cada trabajador reclutado, o bien, un monto proporcional al volumen de trabajo realizado; y establecen contratos en muchos casos verbales (Vaneckere, 1988).

El intermediario tradicional difiere de otros agentes o instituciones que pueden desempeñar una o más de esas actividades, tales como asociaciones de productores, sindicatos y agencias estatales, de las que se distinguen centralmente por su orientación y 
estilo de operar. Tampoco se les debe confundir con las grandes agencias contratistas, que funcionan con una lógica netamente empresarial (empresas vendedoras de fuerza de trabajo eventual), pero con las cuales algunos intermediarios tradicionales pueden estar vinculados como eslabones subordinados para el reclutamiento de trabajadores en las regiones de expulsión de los jornaleros migrantes.

En México, su presencia está reconocida en polos de atracción de diferentes escalas, movilizan pequeños o grandes contingentes de trabajadores solos o acompañados de sus familias y, por lo ya expuesto, se asocian con las redes y circuitos de trabajo migratorio. Los mercados laborales a los que tienen acceso pueden estar dominados por pequeños y medianos productores, como en el caso de Morelos, o bien por grandes empresas agrícolas, como las agroexportadoras hortofrutícolas del noroeste del país.

Para Latinoamérica, el papel de estos agentes contratistas en la movilización y control de la fuerza de trabajo ha sido analizado fundamentalmente desde su dimensión económica, como enlaces entre el capital y el trabajo, destacando su importancia en el desarrollo del capitalismo agrario, toda vez que aseguraron las fuentes de abastecimiento de mano de obra y regularon con cierta eficacia la oferta y demanda de trabajo (Vilar y Samaniego, 1981; Sánchez, 1992; Montes, 1994; Marañón, 2000). Históricamente, el surgimiento de intermediarios laborales ha estado relacionado con la expansión de cultivos intensivos de trabajo que llevaron a los empleadores a promover la formación de fuerza de trabajo apropiada, para satisfacer una relativa escasez de mano de obra en determinados momentos del ciclo agrícola (Vilar y Samaniego, op.cit). ${ }^{1}$ Por tanto, su evolución con frecuencia forma parte del proceso de formación de mercados de trabajo migratorios, donde la búsqueda de trabajadores disponibles a considerable distancia fue resultado de la combinación de una alta demanda estacional y una oferta impredecible de mano de obra temporal (Balán, 1980).

Algunos autores sostienen que los medios coercitivos de los viejos sistemas de enganche cayeron en desuso ante la consolidación del jornalero agrí- cola como sujeto social, a la monetarización de la economía campesina tradicional y la ampliación de las comunicaciones, entre otros factores. Incluso, se argumenta que la integración regional de los mercados de trabajo, la modernización de las empresas agrícolas y/o el desarrollo de asociaciones de productores y sindicatos, fomentaron la contratación directa y relaciones más contractuales, haciendo a los intermediarios tradicionales prescindibles $\mathrm{o}$, al menos, en proceso de decadencia (Vilar y Samaniego, op.cit.; Montes, op.cit.).

Sin embargo, estos puntos de vista no coinciden con la evidencia proporcionada por diversos estudios realizados en México (Paré, 1987; Vaneckere, 1988; Torres, 1994; Lara, 1996; Barrón, 1997 entre otros) y Estados Unidos (Mines y Anzaldua, 1982; Kearney, 1986; Martin, 1989; Villarejo y Runsten 1993; Zabin 1992; Zabin, et.al. 1993). Por contrario, en esa literatura se refleja que los intermediarios tradicionales no sólo no han perdido vitalidad, sino que en algunos casos han reforzado y expandido su influencia en diferentes regiones agrícolas, tanto en dinámicos mercados de trabajo ligados a los cultivos hortofrutícolas, como en zonas menos desarrolladas de producción de hortalizas, caña, café y tabaco. Los intermediarios tampoco han dejado de usar elementos coercitivos extraeconómicos, no siempre visibles y, muchas veces, sustentados en lazos de parentesco, paisanaje y patronazgo. Las amenazas de despido, la retención de salarios, el cobro de cuotas para condicionar el acceso al empleo y otros medios de presión sobre los trabajadores, son algunas de las formas directas en que los actuales intermediarios mantienen disciplinada a esa fuerza de trabajo eventual. Asimismo, algunos autores han mencionado, el rol activo de los sistemas de intermediación en generar mercados "cautivos" de mano de obra, propicios para las grandes empresas agrícolas (C. de Grammont, 1992; Lara, 1996). En fin, los intermediarios laborales no sólo facilitan la imposición de regímenes informales e intensivos de explotación, sino también la reducción del costo de la fuerza de trabajo para el empleador, toda vez que asumen o delegan a los propios jornaleros parte de sus costos de reproducción.

\footnotetext{
${ }^{1}$ Para América Latina, los antecedentes del sistema de enganche se remontan a distintos mecanismos de endeudamiento de trabajadores a fines del siglo XIX, cuando el auge de la exportación de bienes primarios al mercado mundial, generó una amplia demanda de trabajo en ciertas regiones (Vilar y Samaniego, op.cit.).
} 
Sin embargo, la clara evidencia de su papel instrumental al servicio del capital ha opacado considerar el carácter ambiguo de su lugar como representantes de los trabajadores. En efecto, la literatura al respecto revela de modo recurrente que los intermediarios también participan como fuentes de crédito de los jornaleros, en su alojamiento cuando están desempleados, como intérpretes y otros varios servicios. Estas funciones son muy significativas cuando se trata de trabajadores vulnerables por su condición migratoria y/o cultural, para quienes el intermediario laboral -parafraseando a Lloyd Fisher (1953)- puede ser un débil, pero a veces, el único apoyo en un medio ajeno y hostil.

Pero ¿puede defenderse a los intermediarios cuando propician la violación de la legislación laboral, cuando inhiben u obstaculizan el desarrollo de sindicatos? En realidad no se trata de argumentar a favor de estos personajes, sino de repensar las relaciones de poder que determinan su comportamiento $\mathrm{y}$, a fin de cuentas, de comprender las condiciones estructurales que posibilitan su mediación entre diferentes sujetos sociales.

\section{MEDIADORES E INTERMEDIARIOS CULTURALES}

Para avanzar en esta perspectiva he considerado útil recurrir al el concepto de mediador o broker propuesto por Erick Wolf $(1967,1976)$ y desarrollado por diferentes autores. Es conveniente detenerse un poco en esta categoría.

De manera sintética se puede afirmar que el mediador o broker es aquel individuo que sirve de vínculo, intercambio y articulación de grupos económica, política, social y/o culturalmente distintos, con la función primordial de ajustar y compaginar esas diferencias. El broker puede jugar múltiples roles como enlace, traductor, negociador, representante, etc. en esos espacios fronterizos, sin suprimir los conflictos, pero mediando entre ellos (Wolf, 1976: 66). Son como amortiguadores necesarios para que los grupos en contacto (incluso contacto antagónico) desarrollen cierto tipo de intercambios económicos, políticos o simbólicos.

Para desempeñarse como tales, los mediadores deben servir al mismo tiempo a los intereses de los grupos entre los que operan. Además, como no pue- den resolver del todo los conflictos -porque si lo hicieran dejarían de servir a su propósito-, generan un espacio contradictorio y ambiguo que, por ende, es muy dinámico (ibid). Ello explica que los mediadores no sean agentes pasivos o neutrales y participen activamente en la estructura de poder (Tapia, 1992).

Por lo expuesto, un genuino broker rebasa el intercambio circunstancial de bienes y servicios, pues su papel debe ser crucial en la interrelación de estructuras sociales básicas del sistema en cuestión y desempeñarlo con carácter de exclusividad (Silverman, 1965). Es decir, un broker ejerce el monopolio forzoso de los canales de acceso a determinados recursos estratégicos, lo que le brinda la posibilidad de perpetuarse en su condición de mediador y de obtener los beneficios que esta actividad ofrece.

Para poder ocupar este lugar y función es necesario contemplar otra característica fundamental del broker: su modo de interactuar. Éste consiste en el predominio de las relaciones informales y habilidad para adoptar patrones apropiados de conducta pública, que siempre está normada culturalmente. Es decir, opera en el contexto de redes sociales informales entendidas como un conjunto de relaciones diferenciadas (compadrazgo, parentesco, amistad, complicidad, vecindad) que posibilitan y sancionan la intermediación (De La Peña, 1993).

Ahora bien, otra vertiente de análisis ha desarrollado la noción específica de intermediario cultural para caracterizar a múltiples y disímiles sujetos sociales que, a lo largo de la historia, han desempeñado papeles como nexo entre dos o más lenguajes, códigos, tradiciones y culturas (Asseo, 1978; Chartier, 1978; Molino, 1978; Venard, 1978). Entonces, considerando las restricciones ya expuestas sobre la categoría de mediador o broker, estos estudios aportan elementos para afirmar que su posibilidad de existencia pasa por la distancia y contacto en ejes que pueden ser: cultura dominante/culturas subalternas, cultura erudita/cultura popular; cultura nacional/minorías étnicas, escritura/oralidad, interior/exterior, etc. Lejos de constituir meras correas de transmisión de intereses y orientaciones en una u otra dirección, los intermediarios culturales son agentes ideológicos que tienen su propia creatividad (Asseo, op.cit.), lo cual les confiere un papel protagónico en la construcción, reproducción y apropiación del sentido en el campo de interacción en que actúan. 
En su estudio sobre la educación bilingüe y las relaciones interétnicas en México, Vargas (1994) ha enriquecido estos planteamientos permitiéndonos generalizar que en ciertos contextos en los que una minoría étnica se relaciona con la sociedad mayor (bajo condiciones de desigualdad económica, social, cultural y política) se generan condiciones objetivas para la existencia de espacios de mediación que, en el plano ideológico en particular, condensan representaciones y prácticas centrales de la comunicación interétnica.

En suma, a la luz de estos planteamientos se sostiene que la categoría de mediador o broker, ofrece un alto valor heurístico para comprender la complejidad de los intercambios entre jornaleros, empleadores e intermediarios en el mercado de trabajo rural. Sobre todo si consideramos que en nuestro estudio particular era indispensable tomar en cuenta las relaciones étnicas entre los distintos sujetos involucrados y, al mismo tiempo, la condición migratoria de los jornaleros como se verá enseguida.

\section{LOS CAPITANES DE TENEXTEPANGO, MORELOS}

Los siguientes apartados se basan en un estudio de caso realizado a fines de la década de los noventa en la localidad de Tenextepango en Morelos, sobre los capitanes, conocidos intermediarios tradicionales en las cosechas del cultivo de frijol ejotero (judía) en esa región (Sánchez, 2006). En años recientes este escenario ha sufrido algunas transformaciones, derivadas sobre todo del aumento de la producción y de los canales de comercialización, que han hecho más compleja la relación entre los diferentes agentes involucrados; sin embargo, tales cambios no implicaron la desaparición del sistema de intermediación laboral mencionado, por lo cual creemos que en lo fundamental nuestros principales hallazgos siguen siendo vigentes.

\subsection{Breve descripción de un microcosmos}

El sistema de producción comercial de hortalizas en los valles irrigados del sur de Cuautla, en el estado de Morelos, se articula en base a grupos sociales diferenciados que detentan recursos estratégicos distintos (tierra, trabajo, dinero, transporte y colocación en el mercado), gran parte de los cuales son de origen extrarregional. Los principales productos cultivados en la región son: ejotes (judías), elotes, calabazas, pepinos y cebollas, destinados en su mayoría al mercado nacional.

La actividad hortícola se sustenta en una gran masa de pequeños productores (ejidatarios, comuneros y pequeños propietarios), que trabajan en parcelas menores a cinco hectáreas, con escaso o nulo financiamiento, tecnología limitada y sometidos a un mercado muy dinámico y altamente monopolizado, en cuya cúspide se encuentran grandes intermediarios comercializadores de productos frescos (García, 1993). Pese a la modesta escala de cada productor, en conjunto este sector contribuye de manera importante en el mercado interno de productos frescos. ${ }^{2}$ También hay que aclarar que esos pequeños productores trabajan individualmente y no existen cooperativas $\mathrm{u}$ otras asociaciones de producción $\mathrm{o}$ comercialización.

Entonces como ahora, la producción inicia su circuito de mercado a través de empresas transportistas (denominadas oficinas), que tienen contacto con bodegueros y comisionistas, principalmente de la Central de Abastos de la Ciudad de México. Su clientela de productores depende de la eficiencia para recoger y transportar la producción cosechada en lo inmediato, ya que no cuentan con infraestructura adecuada para almacenarla. A fines de los noventa eran alrededor de una docena de empresas de tamaño variable que controlaban los canales de acceso de los horticultores al mercado nacional. Cabe notar que la mayoría de los empresarios transportistas eran mestizos inmigrantes, lo cual condicionaba su posición en el ámbito local.

En el caso particular de la cosecha del ejote (judía), la expansión de la producción incidió directamente en la conformación de un mercado estacional

\footnotetext{
${ }^{2}$ Por ejemplo, la producción de judías de Morelos, desde los años setentas, ocupó el segundo o tercer lugar en la producción nacional de esa hortaliza y, desde el 2003, se colocó en el primero.
} 
que ha sido polo de atracción para miles de jornaleros migrantes. ${ }^{3}$ Estos jornaleros debían laborar en muchas huertas dispersas en la región del oriente de Morelos, e incluso del vecino estado de Puebla.

En el periodo estudiado, el mercado de trabajo estacional representaba un volumen estimado de 2,500 a 3,000 personas, en su mayoría ( $80 \%$ ) provenientes del estado vecino de Guerrero (Sánchez 1996; PRONJAG 1997). Esta población flotante se ha establecido en el pueblo de Tenextepango, entre noviembre y abril, si bien el tiempo promedio de estadía de cada trabajador suele ser menor (alrededor de 2.5 meses).

Los jornaleros temporales en el corte del ejote son jornaleros-campesinos de una región pluriétnica, la Montaña de Guerrero, donde existen comunidades nahuas, mixtecas y tlapanecas que tienen condiciones desfavorables para mantener la agricultura de subsistencia y, por lo mismo, la alternan con una creciente participación en diversos circuitos migratorios. ${ }^{4}$ Para el típico migrante estacional este trabajo en Morelos era el único ingreso monetario significativo, ya que las oportunidades de empleo en sus comunidades han sido escasas y peor remuneradas. Es característico que se trata de una migración familiar, donde sus diferentes miembros participan en el trabajo asalariado, pues se paga a destajo, y los niños laboran desde temprana edad. No ha existido ninguna estabilidad en el empleo y se les asignan precarias viviendas, donde a veces viven hacinadas varias familias. Aunque entre los migrantes estacionales un porcentaje importante son bilingües, sus dificultades en el manejo del español y, sobre todo, su discriminación cultural, marcan su interacción con la sociedad receptora.

Ahora bien, Tenextepango es una comunidad mestiza y urbanizada, cercana a la ciudad de Cuautla, que hacia fin de siglo tenía alrededor de $8 \mathrm{mil}$ habitantes. Parte de su población (10\%) estaba constituida por inmigrantes residentes de Guerrero y Oaxaca que se dedicaban a las labores agrícolas como peones asalariados. Como se puede apreciar en estas cifras, la presencia fluctuante de miles de trabajadores temporales durante la temporada de cosecha, no podía pasar desapercibida en la vida de esta comunidad morelense.

Tenextepango se erigió en centro de operaciones de la actividad hortícola regional, no sólo porque constituía un espacio especializado de compra-venta de fuerza de trabajo eventual, sino además porque en ese lugar se establecieron las empresas transportistas ya mencionadas.

La movilización de la fuerza de trabajo y los sistemas de contratación se adecuaron a ritmos dinámicos de demanda y expulsión de jornaleros, de acuerdo a las características particulares de cada cultivo hortícola. En el caso de la cosecha ejotera, el reclutamiento y organización de la fuerza de trabajo ha reposado exclusivamente en los intermediarios conocidos como capitanes. ${ }^{5}$ Éstos, también inmigrantes radicados en Tenextepango, conforman cuadrillas de tamaño variable -desde unas decenas hasta más de cien personas-, compuestas de migrantes estacionales y, en menor medida, de peones locales -autóctonos e inmigrantes.

En síntesis, consideramos que había un contexto favorable al surgimiento y desarrollo de espacios de mediación, dada la distancia estructural de los diferentes grupos y sectores sociales a recursos que son considerados estratégicos. El control de la tierra y del agua están en manos de los productores lo que les permite negociar su participación en las ganancias que el sistema genera; para lograr alguna rentabilidad (o, al menos, costear su inversión y seguir activos), éstos requieren explotar mano de obra barata y eventual, así como acceder oportunamente a los canales de comercialización de bienes primarios fuera de la región. Por su parte, los tra-

\footnotetext{
${ }^{3}$ En cambio, los restantes cultivos mencionados han sido cubiertos con mano de obra familiar y asalariada en el ámbito local. Hay que decir que el monto del trabajo familiar es poco significativo en relación al trabajo asalariado, no sólo por las características de los cultivos, sino también porque las familias productoras tienden a diversificar sus fuentes de ingreso, prefiriendo otras alternativas ocupacionales complementarias.

${ }^{4}$ El porcentaje mayor de los migrantes trabajan como jornaleros agrícolas en distintos estados del país, pero también hay desplazamientos con destino urbano y migraciones interncionales a Estados Unidos.

${ }^{5}$ Para el resto de las actividades agrícolas de otros productos las formas de contratación son más flexibles, incluyendo la contratación directa y a través de pequeñas cuadrillas en las que uno de los trabajadores se erige como representante para negociar las condiciones de contratación con los productores.
} 
bajadores, en particular los jornaleros migrantes estacionales, conforman el sector más desfavorecido en el sistema, y aunque el ingreso que perciben sea mínimo, éste es crítico para asegurar su sobrevida como campesinos en sus comunidades de origen o para complementar sus ingresos anuales como proletarios agrícolas itinerantes. Por último, los empresarios transportistas, los comisionistas en la Central de Abastos y los capitanes constituyen diferentes clases de intermediarios que, colocados en puntos clave de la cadena de producción y circulación de las mercancías, obtienen su propio beneficio de la explotación de hortalizas desarrollada por pequeños y medianos productores.

\subsection{Relaciones interétnicas y situación de contacto}

Es necesario subrayar que, entendido como un campo de interacción específico (Bourdieu, 1990, 1991), los lugares ocupados por productores, intermediarios comerciales, intermediarios laborales y jornaleros están, al mismo tiempo, sobredeterminadas por el origen étnico y condición migratoria de sus ocupantes, lo cual permea y se traduce en posiciones diferenciadas que expresan relaciones de poder económico, político y cultural. ${ }^{6}$

La interacción entre individuos y grupos que participan en este microcosmos social constituye un espacio complejo de relaciones de diversa índole (económica, social, cultural y simbólica) que determinan las acciones, expectativas y posibles trayectorias de cada integrante. En estas coordenadas, la condición étnica constituye un principio de identificación y diferenciación fundamental. Así, el encuentro entre mestizos e indígenas puede ser visto como una situación de contacto interétnico (Cardoso, 1992: 30-31), donde la admisión implícita de una jerarquía de status (o un sistema de estratificación), ocurre paralelamente al reconocimiento de una estructura de clases del sistema social inclusivo, en la que la naturaleza de las relaciones entre mestizos/locales e indígenas/foráneos es de dominación y sujeción.

Por razones de espacio no es posible ahondar en las múltiples formas en que se manifiesta esta situación de contacto y el conjunto de representaciones que cada grupo étnico elabora acerca de ello. Sin embargo, para los fines de esta exposición, lo que interesa resaltar es que la distancia e interdependencia entre jornaleros estacionales y comunidad local produce un juego dialéctico entre dos tendencias: por un lado, la constante discriminación de los indígenas y la dinámica de trabajo que lleva a los migrantes a permanecer la mayor parte del tiempo en las huertas (fuera del pueblo), generando condiciones para mantener las diferencias culturales de cada grupo; y por otro, la necesaria producción y reproducción de códigos y valores congruentes o comunes que, reduciendo las diferencias, permiten su interacción (Barth, 1976: 18).

Así, esta complementariedad entre jornaleros y productores evidentemente no hace menos asimétrica la relación, ni salva la dificultad que unos y otros encuentran para entablar un intercambio de bienes y servicios de modo directo; tales obstáculos son percibidos por los diferentes actores como resultado de las barreras lingüísticas y culturales entre indios y mestizos.?

En las siguientes páginas trataremos de dar elementos para mostrar cómo, justamente, es en la dinámica de tales intercambios entre grupos culturalmente diferenciados que los capitanes se han conformado, de uno u otro modo, en la pieza clave que permite la interlocución y la negociación entre ambos.

\subsection{Los capitanes como mediadores}

Los servicios que los intermediarios laborales prestan a los productores en la cosecha del ejote son básicamente los siguientes: reclutamiento; supervi-

\footnotetext{
${ }^{6}$ Recuperamos de Michel Pecheux la distinción entre lugares y posiciones en la estructura social, donde los primeros representan un conjunto de rasgos objetivos característicos (por ejemplo, productor/empleador/mestizo/autóctono, o bien trabajador/jornalero eventual/indígena/migrante), y los segundos corresponden a la representación que de esas situaciones se hacen los sujetos y que operan como formaciones imaginarias en el proceso discursivo (Pecheux, op.cit.:48).

${ }^{7}$ Los productores agrícolas en su calidad de mestizos autóctonos dicen no entender a los indígenas fuereños, no saber manejarlos ni asegurar su permanencia en los campos, si no es con la ayuda de los capitanes. Los jornaleros, por su parte, buscan al capitán que los conoce y los trata bien, que sabe hablar en su propia lengua, que respeta a su familia y les apoya en diferentes aspectos.
} 
sión directa del trabajo (incluyendo la productividad y control de calidad); pago a los jornaleros; alojamiento a los trabajadores migrantes y sus familias; envasado del producto; coordinación del transporte y entrega al transportista, así como otros servicios complementarios.

El prestigio de cada capitán es determinante en la construcción de su clientela de productores y está basado en la responsabilidad, oportunidad y calidad de su trabajo, a la vez que en el manejo de cuadrillas eficientes y disciplinadas; por su parte, los empleadores tienen la obligación de pagar al término de la venta de su cosecha, los servicios del capitán y el trabajo efectuado por sus peones.

Por otra parte, la posibilidad de contratación para cada jornalero pasa necesariamente por ser miembro de una cuadrilla dirigida por un capitán. Entonces, aunque los productores sean los empleadores demandantes de esa mano de obra, desde el punto de vista de los jornaleros, el "patrón" que le da trabajo y paga su salario es el capitán.

En ausencia de contratos formales, cada trabajador tiene la posibilidad de cambiar de cuadrilla cuando lo estime conveniente, en función de asegurar continuidad en el trabajo para él y su familia. ${ }^{8}$ La relativa estabilidad que se presenta en la relación entre capitanes y cuadrillas depende de las prácticas de reclutamiento y, en general, del compromiso informal que se establece acerca de las condiciones de trabajo y otros servicios que están implícitos.

En efecto, los capitanes cumplen funciones de gran importancia para los migrantes estacionales, particularmente el traslado desde sus comunidades y el alojamiento, pues muchas familias no podrían sufragar estos gastos por su cuenta. Estos mecanismos permiten a cada intermediario nuclear trabajadores y garantizar el cumplimiento de sus contratos con los productores. Ello no invalida que exista un flujo permanente de trabajadores que circulan entre una cuadrilla y otra, debido a la discontinuidad en el empleo, los bajos salarios y la competencia entre capitanes. La tolerancia de los capitanes hacia esta práctica de autoreclutamiento de los trabajadores, expresa uno de los mecanismos que en este mercado de trabajo permite regular la demanda y el suminis- tro de mano de obra en aquellos momentos y lugares donde se intensifica o decrece la actividad.

Adicionalmente, el capitán proporciona apoyos de diversa índole que sobrepasan el ámbito estrictamente laboral, que no se hacen explícitos en el contrato verbal con los trabajadores, pero que son críticos para la relación. En efecto, a cambio de lealtad y eficiencia, los trabajadores reciben bienes y servicios que les permite reducir los costos de su estadía en Tenextepango y mitigar su condición transitoria, precaria y de marginación cultural. El ejemplo más evidente es el crédito que muchos capitanes brindan como recompensa a los trabajadores que han ganado su confianza en el trabajo. También es significativa su ayuda y/o gestión de recursos en casos de accidentes y enfermedades de los jornaleros a su cargo; o bien, interviniendo en favor de éstos en la resolución de litigios entre trabajadores o con personas de la comunidad local. En todos estos casos el papel del capitán es clave como traductor entre lenguas indígenas (nahua, mixteco o tlapaneco) y el español, dentro y fuera del lugar de trabajo. Esa condición de intérprete no se limita al aspecto lingüístico, ya que su desempeño como representante y negociador supone un amplio dominio de los valores y costumbres de mestizos e indígenas.

Esta relación básica entre empleadores, intermediarios y trabajadores agrícolas se hace más compleja por el rol que desempeñan los empresarios transportistas, con quienes los capitanes mantienen un estrecho intercambio de servicios. Éste consiste, en lo esencial, en que estos últimos se comprometen a prestar sus vehículos para transportar a los jornaleros (desde sus comunidades de origen y a nivel local entre las huertas), a cambio de lo cual los capitanes tienen la obligación de que sus clientes-productores transporten el producto en la empresa que brindó aquellos servicios. Aún cuando el trato entre empresarios transportistas y productores no depende únicamente de la relación de unos y otros con los capitanes, sí entra en el campo de influencia de estos intermediarios asegurar prontitud y calidad del servicio prestado, así como intervenir en el papel de conciliadores en eventuales discrepancias. Este aspecto es fundamental dado que el carácter perecedero del

\footnotetext{
${ }^{8}$ A diferencia del sistema de enganche tradicional, en este caso los trabajadores no reciben pago por adelantado y, en rigor, el trato entre jornalero y capitán puede ser rota por ambas partes al término de cada jornada de cosecha.
} 
producto, impone un margen estrecho para el trabajo de recolección y transporte del mismo al mercado.

De esta manera, los diferentes grupos sociales involucrados en esta actividad económica han delegado en los intermediarios aspectos críticos de la negociación de sus respectivos intereses, lo que permite la coordinación y complementariedad de racionalidades distintas. Si el poder esencial de los capitanes emana del control de los canales de acceso al recurso trabajo, el modo en que operan -reforzando un intenso intercambio de bienes y servicios en distintas direcciones-, trasciende el plano estricto de compra-venta de fuerza de trabajo. Es precisamente por su situación y relaciones con las demás posiciones del campo en cuestión, que los intermediarios laborales pueden potenciar el valor relativo de su poder de enlace e intercambio económico y convertirse en salvaguardas del juego en su conjunto.

Para comprender plenamente su condición de broker o mediador, es necesario conocer algo más acerca de quiénes son los capitanes y cuáles sus recursos.

\subsection{Trayectoria y recursos de los capitanes}

Existían en la comunidad de Tenextepango alrededor de veinte capitanes. Todos inmigrantes con cierta antigüedad en la zona; la mayoría originarios del estado de Guerrero, pero también de Oaxaca, Puebla e Hidalgo. Comenzaron como trabajadores estacionales $\mathrm{y}$, al cabo de dos o más temporadas agrícolas, se establecieron en forma definitiva, a la vez que fueron ascendiendo en la escala ocupacional hasta alcanzar su posición como intermediarios. Por lo general no poseen tierras, si bien algunos las rentan o trabajan a medias con algún productor. $\mathrm{Su}$ actividad como capitanes se alterna con el trabajo en otras labores agrícolas especializadas -y mejor pagadas-, que se realizan entre una cosecha y otra.

Para coordinar y realizar las distintas tareas en el manejo de sus cuadrillas, todo capitán forma un equipo de trabajo compuesto de dos clases de ayudantes: apuntadores y envasadores. Los primeros son los responsables directos de registrar el trabajo realizado por cada peón y calcular su pago (es requisito que sean alfabetos). Los envasadores, como su nombre indica, se encargan de envasar el producto al pie del huerto en costales de 60 kilos. Pero además, unos y otros realizan diversas tareas menores que facilitan las funciones de planificación y control de los capitanes, para lo cual tienen que estar a su disposición. Estos equipos de trabajo representan una estructura especializada, jerarquizada, relativamente estable compuesta por individuos que son de procedencia indígena, inmigrantes o estacionales.

Desde el punto de vista de los procesos de mediación, en sentido amplio, esta pequeña estructura está equipada social, lingüística y culturalmente para ajustar e integrar los intereses de los jornaleros y los productores en el proceso técnico de trabajo y, sobre todo, representar y manipular los intereses específicos de ambos grupos.

Por tanto, habría que ampliar la imagen del broker como individuo - el capitán-, a la del equipo: capitán y ayudantes. Además, la manera en que esta estructura especializada puede desempeñar esas funciones depende, en gran medida, en cómo se genera y reproduce a sí misma.

De acuerdo a la evidencia, las relaciones entre el capitán y sus ayudantes son de carácter asimétrico, y la distancia social es mayor en el caso de los envasadores. En muchos casos el equipo se crea a partir de un campo de relaciones simétricas - entre compañeros de trabajo, compadres o miembros de una red de intercambio recíproco -, que con el tiempo se convierten en relaciones de tipo patrón-cliente. ${ }^{9}$

El envasador tiene un papel clave como nexo con las comunidades proveedoras de mano de obra. Las dos modalidades básicas son: a) se trata de un inmigrante que controla toda una red de parentesco en su comunidad de origen y viaja cada año a reclutar a sus propios paisanos; o bien, b) es un migrante temporal que es reconocido como representante de su grupo (también una red parental) para establecer trato con un capitán determinado. El capitán, por su parte, ofrece un trato preferencial al contratarlo como envasador, pues su labor es menos pesada y percibe un sueldo semanal fijo. En ambas modalidades, el grupo de jornaleros migrantes puede identi-

\footnotetext{
${ }_{9}^{9}$ Dicha trayectoria coincide con los procesos de intermediación analizados por Claudia Pedone (2005) para explicar la relación de las redes migratorias y la verticalización de sus recursos en su articulación con el mercado de trabajo agrícola en Murcia.
} 
ficar al envasador como un intermediario entre ellos y el capitán, y considera que la posición de aquél le asegura cierta estabilidad laboral en esa cuadrilla. De esta manera, se genera una cadena de intermediaciones compleja desde las comunidades emisoras de jornaleros y los productores, que son quienes pagan por el trabajo realizado.

Este hecho, además, no excluye que cuando los capitanes son indígenas guerrerenses tengan vínculos con redes migratorias en sus comunidades de origen de las que se sirven para abastecer sus cuadrillas y que se complementan con este rol de los envasadores.

En suma, la cohesión interna y capacidad de reclutamiento del capitán y su equipo operan en el marco de un sistema de lealtades personales: el parentesco, el compadrazgo, la reciprocidad entre vecinos y el origen geográfico común. Así puede entenderse que las prácticas de reclutamiento y control social sobre los trabajadores condicionan la composición misma de las cuadrillas, donde se registra una clara presencia de amplios núcleos de trabajadores emparentados de una comunidad determinada o comunidades vecinas de igual filiación lingüística.

En cuanto al propio capitán, en su condición de mestizo o de indio asimilado a la cultura local, constituye el interlocutor legítimo para productores y transportistas. Como "hombre de campo", el capitán se identifica con el productor, pero al mismo tiempo, ha vivido en carne propia las necesidades de los peones. En menor medida, como fuereño y pequeño empresario se representa a sí mismo, al igual que el transportista, como parte de los inmigrantes que han dado prosperidad económica a la agricultura regional.

La trayectoria personal del capitán revela el proceso mediante el cual ha ido construyendo una red de relaciones diferenciadas con productores, transportistas y trabajadores, adquiriendo a su paso la experiencia para desenvolverse entre mundos distintos y manipular de manera juiciosa las necesidades y expectativas de cada uno. Dentro del equipo, sobre el capitán recae la mayor responsabilidad en adoptar patrones apropiados de conducta pública que posibilitan y sancionan la intermediación. En el momento crítico de la cosecha, el capitán debe refrendar su capacidad para cumplir las funciones a él delegadas por unos y otros. Su legitimidad está en cuestionamiento permanente y debe velar por reproducir su propio dominio, con lo cual reproduce el equilibrio en el sistema.

A diferencia de los empresarios transportistas o los bodegueros, los capitanes no cuentan con capital económico significativo y menos aún con poder político a nivel local. En contrapartida a su escaso capital económico y político, los capitanes cuentan con otro tipo de recursos: capital social y capital cultural (Bourdieu, 1991). Tales recursos les han dado cierto prestigio y autoridad en la región de Cuautla en Morelos y en la región de la Montaña en Guerrero, si bien no han permitido que accedan a instancias clave de la toma de decisiones. Los productores de Morelos han tenido sumo cuidado de evitar que dicho poder se acreciente, por ejemplo, restringiendo la participación de los capitanes en ocasión de asambleas, con la justificación de que no son ejidatarios, no tienen tierras propias y no son nativos.

En consecuencia, sostenemos que el capital fundamental acumulado por los capitanes para desempeñarse como brokers en este microcosmos no es de índole económica, sino social y cultural. Además, este último le permite traducir su poder en capital simbólico (Bourdieu, 1990: 283) pues es el mediador legítimo en la articulación de tres ejes, cruciales en este contexto: agricultura comercial/agricultura de subsistencia; cultura mestiza/minorías étnicas; locales/foráneos.

Esta posición particular hace de los capitanes activos participantes en la construcción, reproducción y modificación de las representaciones sociales de los diferentes actores involucrados. Por un lado, refuerzan el carácter asimétrico de la relación entre el productor-empleador y el trabajador, añadiendo otros medios de subordinación de éste a aquél (y que conforman su ámbito específico de poder como intermediario). Frente a los jornaleros, el lugar del patrón es representado -y justificado- como un productor sometido a los grandes comerciantes de productos frescos, que no puede ofrecer mejores salarios o condiciones de trabajo porque su margen de ganancia es limitado; pero también, aduciendo a la condición de campesinos pobres e indígenas del lugar ocupado por los trabajadores, el intermediario reproduce la inequidad de beneficios como resultado "inevitable" de un estado natural de cosas. Con ello, el intermediario legitima la relación de dominación sobre los jornaleros, basado en estrategias simbóli- 
cas de racionalización y naturalización de esa relación (Thompson, 1993).

Por otro lado, los capitanes están involucrados en el discurso de los migrantes, defendiendo frente a patrones, transportistas y comunidad local, el derecho al empleo de aquéllos. Este derecho no implica sólo la correspondiente retribución al trabajo desempeñado, sino también a la forma en que se realiza, introduciendo los criterios propios de los trabajadores en la distribución y asignación de cargas dentro de las cuadrillas y unidades familiares, así como otras prácticas laborales que no corresponden tanto a las condiciones técnicas de la producción hortícola o a una lógica empresarial, sino a las formas y criterios con que el trabajador ha condicionado su inclusión en el sistema. En ese sentido, el capitán también apela a la naturaleza y tradición de los jornaleros (por ser campesinos e indígenas), en su representación del lugar de los trabajadores, cuestión que es reproducida en su papel de portavoz de los mismos.

Entonces, si bien es cierto que, en última instancia, los capitanes han surgido para resolver las necesidades de la agricultura comercial -en tanto instrumentos de la movilización y control de la fuerza de trabajo para la actividad hortícola-, este hecho no debiera soslayar la importancia de su capacidad de incorporar prácticas sociales de los jornaleros migrantes que tienen que ver con la manera en que las familias jornaleras se apropian de los lugares de trabajo y que alteran la planificación preestablecida por los capitanes y los productores. Al hacerlo, los capitanes no sólo obedecen a las relaciones y lealtades que mantienen con sus equipos y sus peones, sino que por ese medio concentran también poder como mediadores.

Uno de los aspectos que ilustra esta situación se relaciona con el problema de la inestabilidad laboral. Este es un elemento crítico de la mediación, en el sentido de representar una síntesis contradictoria de compromisos, reflejo de la estrecha interdependencia de los diferentes agentes económicos y del carácter conflictivo del lugar y función que ocupan los capita- nes. Es decir, si por un lado las necesidades fluctuantes y dinámicas de trabajo en la cosecha del ejote imponen como característica estructural de ese mercado de trabajo la inestabilidad laboral, desde las prácticas de las familias jornaleras, por el otro lado, dicha inestabilidad ha sido apropiada como flexibilidad de movimiento. Flexibilidad para ir y venir de sus pueblos -aprovechando la relativa proximidad geográfica-, flexibilidad para distribuir y redistribuir sus recursos humanos entre aquí y allá a lo largo de la temporada de cosecha para atender asuntos familiares, asistir a la fiesta patronal o trabajar en la propia milpa. Para los capitanes, dicha volatilidad de los jornaleros es admitida porque la prestación de la vivienda no es un mecanismo de control absoluto, pero además porque difícilmente podría ofrecer ocupación continua a lo largo de la temporada de cosecha. Entonces, debe consentir y planificar sobre la marcha las ausencias temporales de parte de sus peones (que no siempre corresponden con la demanda y ritmo de la cosecha), reconociendo en estas prácticas la facultad legítima de los indígenas de atender sus compromisos sociales y rituales.

En otro sentido, esa flexibilidad de movimiento, aunada a la posibilidad de intercambiar de empleador toda vez que no existen relaciones contractuales, se traduce en un sentimiento de libertad que es positivamente valorado por los jornaleros. Paradójicamente en Tenextepango, el trabajador no es un nombre, es un número,${ }^{10}$ pues no decide para quién, ni cuándo, ni dónde trabajar, sólo le cabe integrarse con su familia a anónimas cuadrillas que son movilizadas por enganchadores de una huerta a otra. Esta situación, entonces, es resistida en el espacio que el sistema permite: el jornalero es libre de asignar tareas dentro de su familia, de enviar alguien al pueblo, de cambiar de enganchador cuando le conviene.

Podrían darse muchos otros ejemplos que reflejan la manera en que los capitanes están obligados a representar a sus trabajadores y de cómo su legitimidad frente a ellos depende de su reputación como abogados en casos de ser acusados por patrones, lu-

\footnotetext{
${ }^{10}$ Nos referimos a la práctica generalizada de control de los peones y su trabajo, que consiste en asignar a cada jefe de familia un "número" que corresponde al orden en que fue registrado como miembro de la cuadrilla. Como el trabajo se paga a destajo, cada jornalero y sus acompañantes se identifican con ese número para que el apuntador anote el monto de kilos de ejote cosechados. Al final de cada semana, el capitán y el apuntador calculan el pago de cada grupo familiar, según la cantidad de kilos acumulados (hayan o no trabajado todos los días en la cuadrilla).
} 
gareños y autoridades, o simplemente de defender que tienen distintas costumbres. ${ }^{11}$

En suma, el ejercicio de poder de los mediadores permea las relaciones de grupo, involucrando el conflicto y el acomodo, construyendo cotidianamente la dominación y la resistencia a la misma.

\subsection{Resumiendo sobre un estudio de caso}

El mercado de trabajo estacional en la cosecha de ejotes en el oriente de Morelos es también un escenario de un intenso contacto intercultural entre una cultura mestiza/local y una cultura indígena/ foránea. En tales circunstancias, se generan condiciones objetivas para que determinados individuos se apropiaran de los canales de comunicación entre niveles social y culturalmente diferenciados.

Los capitanes monopolizan los canales de acceso a la mano de obra y su presencia se ha vuelto parte estructural de la propia dinámica de este mercado de trabajo. Pero, además, la construcción y mantenimiento de un conjunto de vínculos diferenciados que sirven de enlace entre los distintos sujetos sociales -productores, jornaleros agrícolas y transportistas-, los hace una pieza clave en el funcionamiento de la producción y comercialización de hortalizas en la región.

Cabe precisar que en este caso los pequeños productores están sometidos a capitales comerciales extrarregionales, por lo que el empleo de mano de obra barata más que un medio de acumulación extraordinaria, es una forma de contener las presiones financieras que resultan del constante aumento de sus costos de producción (en particular de insumos agrícolas como los agroquímicos), en tanto el mercado siempre condiciona los precios a sus cultivos.

La posibilidad de integrar las prácticas y los discursos de los distintos grupos implica no sólo compaginar el intercambio de bienes y servicios de carácter económico, sino de concertar la permanente negociación de lógicas sociales y culturales disímiles.
La presencia de vínculos de patronazgo en la relación con sus clientelas y su alineamiento a la preservación del status quo en la distribución de poder, no excluye la existencia de códigos de conducta que exigen niveles de complicidad y representación de los intereses de los trabajadores a su cargo. En el contexto local, ello ha representado la posibilidad para las familias jornaleras migrantes de flexibilizar sus estrategias de sobrevivencia y de ampliar las condiciones de reproducción de su identidad cultural.

\section{ALGUNAS REFLEXIONES COMPARATIVAS Y GENERALES}

Las características específicas de los capitanes en el mercado de trabajo del oriente de Morelos, podrían asemejarse a lo que ocurre en otras regiones agrícolas que constituyen mercados laborales etnificados (Pedreño, 2005) y polos de atracción de jornaleros migrantes.

De acuerdo a nuestra experiencia, una condición fundamental para que esos u otros intermediarios puedan reproducirse como mediadores, depende de su posibilidad de mantener su independencia respecto a los empresarios (y al Estado). En el caso de estudio, el tipo de explotación agrícola de la región -atomizada en una multiplicidad de pequeñas unidades productivas-, representa un mercado de trabajo fragmentado y dislocado, que no sólo refuerza la necesidad de intermediación en este ámbito, sino que garantiza la autonomía de los intermediarios respecto a los empleadores agrícolas. Considero que este es un factor clave para entender su especificidad, sobre todo en comparación con otros sistemas de agricultura comercial basados en grandes unidades productivas y pocos propietarios, en donde el margen de maniobra de los intermediarios puede ser distinto.

En segundo lugar, se puede señalar que en el caso analizado es notorio que cada capitán y su equipo controlan toda la cadena de intermediación de acceso al trabajo, cuando en regiones más dinámicas y complejas existen diferentes clases de interme-

\footnotetext{
${ }^{11}$ Cuando realicé este estudio llamó mi atención la complicidad de los capitanes en la extendida práctica de robo de ejotes para revenderlos a acaparadores ilegales. Algunos capitanes manejaban un doble discurso, instando a sus peones a no robar, y al mismo tiempo, alertándolos para no ser sorprendidos.
} 
diarios que pueden especializarse en alguna fase o función: contratistas y apuntadores en el transporte de larga distancia desde las zonas de origen de la mano de obra, "camioneteros" o "furgoneteros" para traslados de corta distancia desde las viviendas temporales a los campos agrícolas, mayordomos u otras figuras en el ámbito de la producción, etc. (por no mencionar, además, la eventual presencia de otras agencias intermediarias públicas o privadas). Estos agentes tradicionales pueden cumplir uno o varios roles en esta cadena sincronizada que se forma desde las zonas de expulsión hasta los lugares de trabajo; en otros casos compiten entre sí o parecieran inducir una mayor segmentación del mercado laboral (por ejemplo, migrantes "enganchados" versus trabajadores que viajan por su cuenta).

A mi juicio, en cada contexto sería importante considerar cuál es el margen de control que tienen los intermediarios sobre el acceso real al empleo, y esto implica el conjunto de condiciones que permiten al trabajador estar en el lugar y momento oportunos para vender su fuerza de trabajo. En ese sentido también habría que tomar en cuenta que los intermediarios laborales, además de movilizar la fuerza de trabajo, deben de inmovilizarla el tiempo necesario, y esto puede significar que aspectos como la vivienda (cuando los trabajadores no pueden resolverlo por sí mismos) pueden ser un recurso crucial en su intercambio con los trabajadores y una fuente importante de poder para sí mismos.

En tercer lugar, nuestra experiencia sugiere que en contextos agrícolas conformados por mercados laborales etnificados (Pedreño, 2005), la posibilidad de brokerage de los intermediarios tradicionales depende en buena cuenta de su pertenencia a las redes migratorias, es decir que sean parte (y resultado) de la experiencia social de la propia migración. En el extremo opuesto, los sistemas intermediarios basados en sujetos locales o donde este tipo de mercados laborales están dominados (o atravesados) por otras instituciones intermediarias públicas o privadas, las relaciones interétnicas entre locales/foráneos, empleadores/trabajadores pueden limitar el lugar y función de los intermediarios laborales como media- dores. En el primer caso, donde los intermediarios emanaron de las propias redes migratorias, es relevante subrayar que la creación de relaciones laborales verticales está oscurecida por lazos de solidaridad y obligaciones informales que atraviesan las fronteras de clase (Krissman, 1994), lo cual torna este vínculo ambiguo, y no por ser contradictorio es poco eficaz para intermediarios y migrantes.

De cualquier manera, la presencia de situaciones de contacto interétnico hace del mercado laboral un espacio privilegiado para la construcción y reproducción de significados, en un juego dialéctico y conflictivo que determina las acciones, expectativas y posibles trayectorias de cada grupo integrante. Representaciones objetivas e imaginarios pueden apelar a repertorios culturales conocidos o recreados a partir de la experiencia sobre los otros, con quienes no había antecedentes de contacto previo al surgimiento de tales nichos laborales. ${ }^{12}$ Este hecho, ligado a las estrategias empresariales que buscan desvalorizar y descualificar la mano de obra (Pedreño, 2005), hace que en tales escenarios laborales haya condiciones propicias para el desarrollo de intermediarios que pueden derivar en brokers que se alimentan de tal vulnerabilidad de la mano de obra y de manipular las fronteras socioculturales con la sociedad receptora. Pero esto no implica que los grupos subordinados tengan un rol pasivo e indefenso, unilateralmente controlado por la producción capitalista, pues pueden desplegar sus propias estrategias de resistencia, apropiarse de sus lugares de trabajo y, en fin, negociar mejores condiciones laborales (Torres, 1994). Si los intermediarios laborales tradicionales pueden o no inclinar la balanza a favor de los trabajadores en ciertas circunstancias, es algo que depende de cada contexto y coyuntura.

En fin, hasta aquí se he tratado de argumentar que entre las complejas facetas de análisis de los mercados laborales agrícolas, es menester explorar el carácter multidimensional de la mediación de los contratistas laborales, de su alcance y límites, en el intercambio de bienes y servicios entre grupos económica y culturalmente diferenciados. Asimismo, creemos que este enfoque permite revisar el espa-

\footnotetext{
${ }^{12}$ Kearney (1986) apunta que la incorporación de jornaleros mixtecos a la agricultura californiana, cuando los intermediarios eran mestizos de origen mexicano, recreó en otro contexto viejas relaciones de patronazgo entre unos y otros, mientras los empresarios estadounidenses no tenían que preocuparse de cómo era disciplinada la fuerza de trabajo.
} 
cio que los intermediarios laborales han construido como enlaces entre los diferentes agentes económicos que intervienen en la actividad agrícola, consti- tuyéndose en elementos protagónicos en la producción y reproducción simbólica de ese conjunto de relaciones sociales.

\section{BIBLIOGRAFIA}

Asseo, H. (1978). "Autor de la notion d'intermediaire culturel”. En Actes du Colloque du Centre Meridional d'Histoire Sociale, des Mentalités et des Cultures, 1978. Aix Provence: Publications de L’Université de Provence.

BALÁN, J. (1980). Migraciones temporarias y mercado de trabajo rural en América Latina. Buenos Aires: CEDES.

BARRón, A. (1997). "Características de los mercados de trabajo de los cultivos no tradicionales de exportación. El caso de las hortalizas en México". En A. Barrón y E. Sifuentes (coords.). Mercados de trabajo rurales en México. Estudios de caso y metodologías. México: Facultad de Economía/UNAM y Universidad Autónoma de Nayarit.

BARTH, F. (1976). Los grupos étnicos y sus fronteras. México: Fondo de Cultura Económica.

Bourdieu, P. (1990). "Acerca de las propiedades de los campos". En P. Bourdieu. Sociología y Cultura. México: CNCA/Grijalvo.

Bourdieu, P. (1991). El sentido práctico. Madrid: Taurus.

Cardoso De Oliveira, R. (1992). Etnicidad y estructura social. México: CIESAS.

C. De Grammont, H. (1992). "Algunas reflexiones en torno al mercado de trabajo en el campo Latinoamericano". Revista Mexicana de Sociología, 1, 49-58.

Chartier, R. (1978). "La culture en question". Actes du Colloque du Centre Meridional d'Histoire Sociale, des Mentalités et des Cultures, 1978. Aix Provence: Publications de L'Université de Provence.

De La Peña, G. (1993). "Poder local, poder regional, perspectivas socioantropológicas". En J. Padua y A. Vanneth (comps.). Poder local, poder regional. México: El Colegio de México/CEMCA.

FisHeR, LL. (1953). The Harvest Labor Market in California. Cambridge-Massachusetts: Harvard University Press.

GARCíA, P. (1993). "Estructura del sector agropecuario y movimientos sociales en Morelos". En U. OSWALD. Mitos y realidades de Morelos. Cuernavaca: CRIM/UNAM.

Kearney, M. (1986). "Integration of the Mixteca and the Western U.S.-Mexico Region via Migratory Wage Labor". En I. ROSENTHAL-UREY. Regional impacts of U.S.-Mexican relations. San Diego: Center for U.S.-Mexican Studies, University of California.

KrisSMAn, F. (1994). Comparing the impacts upon mexican bi-national migrants of incorporation into network-based institutions in California. San Diego: Center for U.S.-Mexican Studies, University of California.

LARA, S. (1996). "Mercado de trabajo rural y organización laboral en el campo". En H. C. DE GRAMMONT (coord.). Neoliberalismo y organización social en el campo. México: UNAM-Plaza y Valdés.

MARAÑón, B. (2000). "Contratistas en mercados hortícolas de exportación : sus funciones económicas". XXII International Congreso of Latin American Studies Association. Miami: LASA.

Martin, P. (1989). California's Farm Labor Market. Davis: California Institute for Rural Studies, Working Paper 4.

MARTíNEZ, J. (2005). Redes sociales, intermediarios y mercado de trabajo rural. Estudio de caso, región centro sur del estado de Morelos, Tesis de Maestría. México: Instituto de Investigaciones Dr. José María Luis Mora.

Mines, R. y R. ANZALDUA (1982). New migrants VS. Old migrants: Alternative Labor Market Structures in the California Citrus Industry. San Diego: Center for U.S.-Mexican Studies, University of California.

Molino, J. (1978). “Combien de cultures?". Actes du Colloque du Centre Meridional d'Histoire Sociale, des Mentalités et des Cultures, 1978. Aix Provence: Publications de L'Université de Provence.

Montes, A. (1994). "Mercado laboral y asalariados agrícolas en la Región de Arequipa". En O. Dancourt, E. Meyer y C. Monge (eds.). Perú. El problema agrario en debate. Arequipa: Universidad Nacional de San Agustín.

PARÉ, L. (1987). "Sindicalización agrícola en la zona cañera del ingenio Emiliano Zapata, Zacatepec, Morelos". En L. Paré, I. Juárez y G. Salazar. Caña Brava. México: UNAM/UAM.

PÊcheux, M. (1983). Hacia el análisis automático del discurso. Madrid: Gredos.

Pedone, C. (2005). "Diversificación de las cadenas migratorias ecuatorianas hacia el mercado de trabajo agrícola de Murcia, España”. En A. Pedreño Y M. Hernández (coords.). La condición inmigrante. Exploraciones e investigaciones desde la región de Murcia. Murcia: Universidad de Murcia.

Pedreño, A. (2005) “Sociedades etnofragmentadas". En A. Pedreño Y M. Hernández (coords.). La condición inmigrante. Exploraciones e investigaciones desde la región de Murcia. Murcia: Universidad de Murcia. 
Pronjag, Coordinacion Estatal Morelos (1997). Modulo de atención para los cortadores de ejote de la región de Cd. Ayala, Morelos. México: SEDESOL.

SÁnchez, L. (1992) “El tránsito perpétuo: los jornaleros migrantes”. Revista Acta Sociológica, 4-5, 143-159.

SÁnchez, K. (1996). Migración de la Montaña de Guerrero: el caso de jornaleros estacionales en Tenextepango, Morelos. Tesis de Maestría. México: ENAH.

SÁNCHEZ, K. (2006). Los capitanes de Tenextepango. Un estudio sobre intermediación cultural. México: Plaza y Valdés/ UAEM.

Silverman, S. (1965). "Patronage and community-nation relationships in Central Italy". Revista Ethnology, 4 (2).

TAPIA, J. (1992). "Intermediación y construcción social del poder en el Bajío Zamorano". En J. Tapia (coord.). Intermediación social y procesos políticos en Michoacán. Zamora: El Colegio de Michoacán.

Thompson, J. (1993). Ideología y Cultura moderna. Teoría crítica social en la era de la comunicación de masas. México: UAM-X.

Torres, G. (1994). La fuerza de la ironía. Un estudio del poder en la vida cotidiana de los trabajadores tomateros en el Occidente de México. Holanda: Universidad Agrícola de Wageningen.

VAnecKere, M. (1988). "Situación de los jornaleros agrícolas en México". Revista Investigación Económica, 18, 171198.

VArgas, M. (1994). Educación e ideología. Constitución de una categoría de intermediarios en la comunicación interétnica. El caso de los maestros bilingües tarascos (1964-1982). México: CIESAS.

Venard, M. (1978). "Sur les intermediaries d' anciens style". Actes du Colloque du Centre Meridional d'Histoire Sociale, des Mentalités et des Cultures, 1978. Aix Provence: Publications de L'Université de Provence.

Vilar, R. y C. SAmaniego (1981). Sistema de contratación y migración laboral temporal en Santa Cruz, Bolivia. La Paz: OIT/FNUAP.

Villarejo, D. y D. Runsten (1993). California's Agricultural Dilemma: Higher Production and Lower Wages. Davis: California Institute for Rural Studies.

Wolf, E. (1967). "Levels of Communal Relations”. En Handbook of Middle American Indians. Austin: University of Texas Press.

Wolf, E. (1976). "Aspects of Group Relations in a Complex Society: Mexico". En T. Shanin (ed.). Peasants and peasant societies. Middesex: Penguin Modern Sociology Readings.

ZABIN, C. (COORD.) (1992). Migración oaxaqueña a los campos agrícolas de California. San Diego: Center for U.S.Mexican Studies, University of California.

ZaBIn, C., et.al. (1993). Mixtecs Migrants in California Agriculture: a New Cycle of Poverty. Davis: California Institute for Rural Studies. 